\title{
Estimation of anti-icing properties of coatings
}

\author{
Valentina Loganina $^{1 *}$, and Svetlana Kislitsyna ${ }^{1}$ \\ ${ }^{1}$ Penza State University of Architecture and Construction, 440028, 28, Street Titov, Penza, \\ Russia
}

\begin{abstract}
The proposed composition for anti-icing coating of metal structures. The composition contains as the filler used aerosil brand R 972 with a density of $2360 \mathrm{~kg} / \mathrm{m}^{3}$, particle size of $16 \mathrm{~nm}$ and a specific surface $12000 \mathrm{~m}^{2} / \mathrm{kg}$. Silicone resin SILRES ${ }^{\circledR}$ MSE 100 with a $10 \%$ concentration was used as a binder. The degree of hydrophobicity was estimated by the value of the wetting angle. In order to characterize the anti-icing properties of the coatings, we used the static and dynamic (advancing and retreating) wetting angle, as well as wetting hysteresis, for which we measured the angles of leakage, and the angles of drift from. Studies of the dynamics of freezing drops on the surface were performed using a TESTO 875-1 thermal imager. It is shown, that the hysteresis of wetting of the superhydrophobic surface based on the developed composition is 3.7 degrees. The critical angle of rolling drops of water from an inclined surface is determined. In the study of the kinetics of freezing of a drop of water on a metal surface, an uneven distribution of temperature on the surface of a drop of water is observed. The process of freezing drops is multistage. In the initial period, there is a transfer of heat from the surface to a drop of water. This stage is followed by the process of freezing the drop, which is manifested in the movement of the freezing front from the substrate upwards.
\end{abstract}

\section{Introduction}

Various anti-icing coatings are used to protect the roofs of buildings against icing. The effectiveness of anti-icing coatings due to their unique properties:

- significant (more than 3 times) slowing down the process of droplet freezing on a super-hydrophobic surface [1];

- the duration of the contact of the superhydrophobic surface with the falling drop is so small that the drop does not have time to freeze [2,3];

- high mobility of liquid droplets on the surface of the coating, which is determined by the small hysteresis of the wetting angle - no more than 10-15 degrees [4,5];

- low adhesion of ice to the surface, due to a small fraction of the wetted surface $(\sim 100$ $\mathrm{kPa}$ ), and anti-corrosion properties [6,7].

The formation of icicles of large size on the edges of roofs of roofs and their subsequent fall can lead to heavy and tragic consequences, not to mention that the fight against this

\footnotetext{
${ }^{*}$ Corresponding author: loganin@mail.ru
} 
phenomenon requires a lot of material costs. Analysis of scientific and technical literature suggests that the production of superhydrophobic materials is possible with a combination of hydrophobic material and surface topography [8]. On the domestic market of anti-icing coatings are presented the compositions "PROL", NIVASAR-300, ULTRA GUARD STOPLED, OS - 12-03, "Slider", "Baltek-Antiice", etc.

Despite the large number of proposed compositions, the problem of anti-icing remains relevant. In some cases, coatings, having a superhydrophobic effect, after a certain period of operation lose the superhydrophobic effect [9-11]. Our studies allowed us to recommend the composition for anti-icing coating containing silicone resin SILRES ${ }^{\circ}$ MSE 100 and aerosil brand R 972 [12]. The results of the studies showed that after wetting for 72 hours a coating based on SILRES ${ }^{\circledR}$ MSE100 silicone resin of $5 \%$ and $10 \%$ concentration retained a superhydrophobic effect - the wetting angle on the mortar substrate was more than 150 degrees. The adhesion of coatings to the substrate, estimated by the method of lattice cut in accordance with GOST 15140-78 "Paint materials. Methods for determining adhesion "after wetting amounted to 1 point.

\section{Materials and research methods}

In this work, we experimentally investigated the dependence of wetting characteristics in the process of spreading drops on the surface of a coating based on the proposed composition. In order to characterize the anti-icing properties of the coatings, we used the static and dynamic (advancing and retreating) wetting angle, as well as wetting hysteresis, for which we measured the angles of leakage, and the angles of drift from.

The dynamic wetting angle was determined by the method of a lying drop. To do this, a drop of water was placed on the surface of the coating using a syringe; the diameter of the drop was 2 to $5 \mathrm{~mm}$. During the measurement of the incurring angle, the needle of the syringe remained in the drop throughout the experiment. Flow angle was measured with decreasing drop size due to suction of water through a syringe (Fig. 1). The difference between the off angle and the flowing angle was determined by the wetting hysteresis.

In addition, a critical angle was determined at which a drop of water from an inclined surface began to roll. To do this, a drop of water was placed on a metal plate, which gradually began to bend. For an experimental study of the process of wetting solid substrates with a liquid, metal sheeting of the "MR-20" roofing sheet with a polymer coating were used as substrates. The composition of the anti-icing coating was applied to the plate, after curing of which a drop of water was applied to the surface. The static wetting angle was determined by the method of a lying drop by the formula

$$
\cos \theta=1-\frac{h}{r}
$$

where $\mathrm{h}$ - is the height of the drop;

$\mathrm{r}-$ is the radius of the drop. 


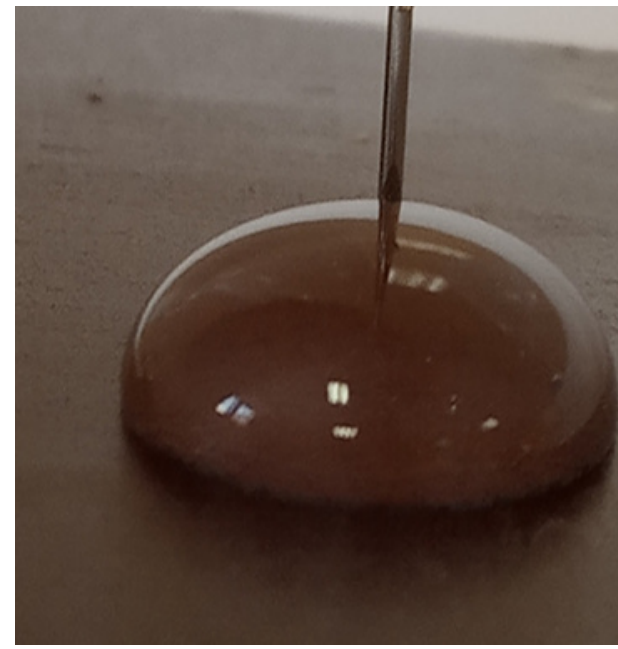

a

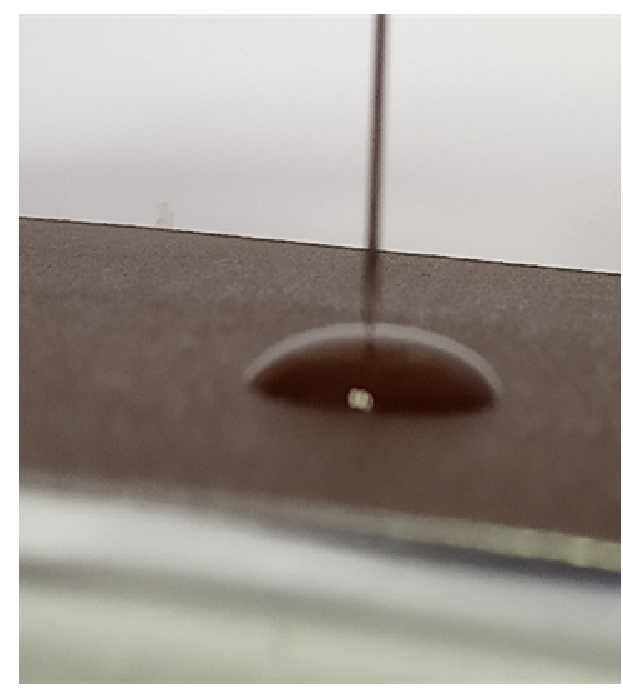

C

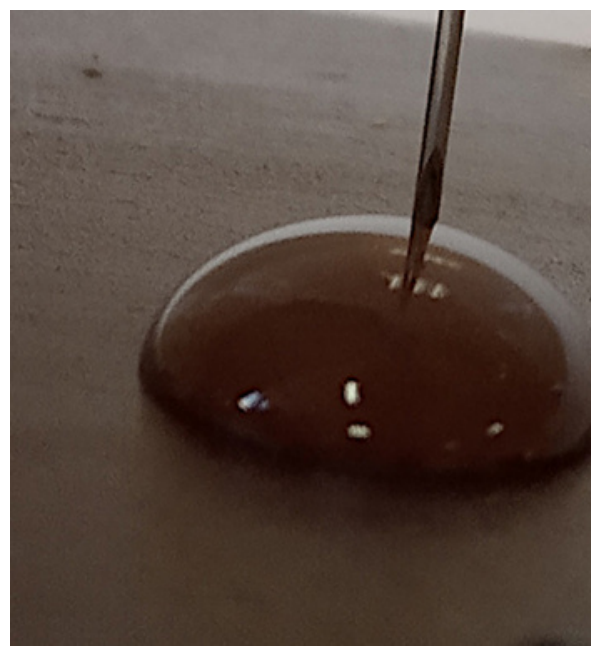

b

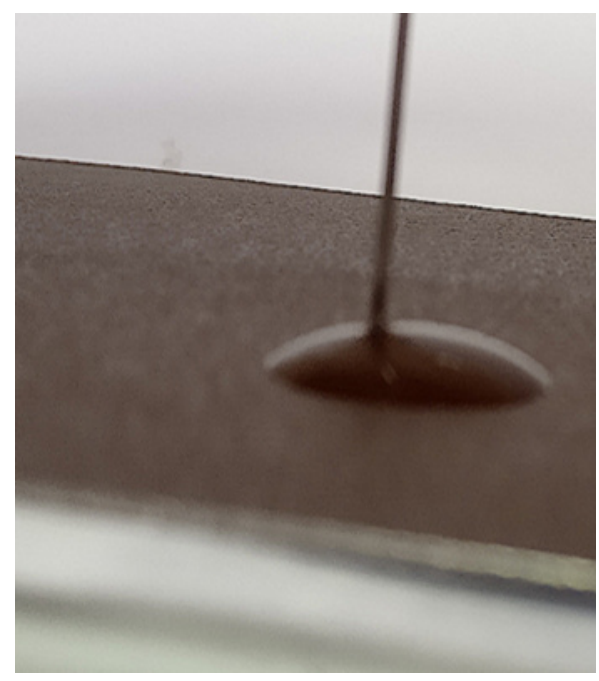

d

Fig.1. Photo of a drop of water on a metal surface a, b - on a superhydrophobic surface; c, d-on a hydrophilic surface; a, c - leakage; b, d - flow

\section{Research result}

The values of angles (Table 1) clearly confirm the presence of hysteresis on the surface. The data are in good agreement with the works [13-15].

When the angle of inclination of the metal plate is $10^{\circ}$, a drop of water from the superhydrophobic surface begins to roll (Fig. 2). Rolling a drop of water on a hydrophilic surface begins at an angle of 31 degrees. 
Table1. Contact angle for water on a metal surface

\begin{tabular}{|c|c|c|c|c|}
\hline Surface type & $\begin{array}{c}\text { Static wetting } \\
\text { angle }\end{array}$ & Off angle & Leak angle & $\begin{array}{c}\text { Wetting } \\
\text { hysteresis }\end{array}$ \\
\hline superhydrophobic & 164 & 162,8 & 166,5 & 3,7 \\
\hline hydrophilic & 25,4 & 21,1 & 45 & 23,9 \\
\hline
\end{tabular}

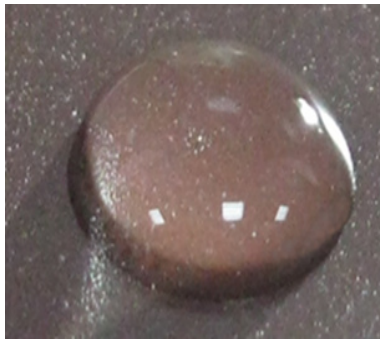

a

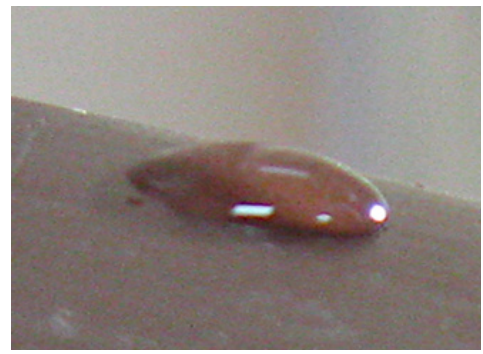

b

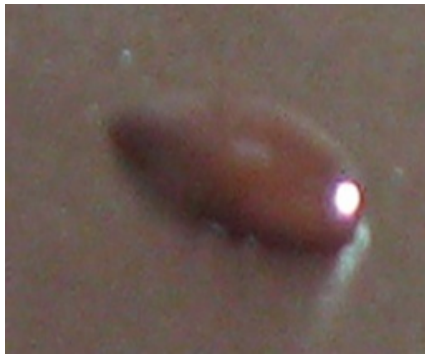

C

Fig.2. Photo of a drop of water on the surface of the anti-icing coating on a metal surface: ain the initial state $\mathrm{b}$ - after 1 second of rolling; $\mathrm{c}$ - after 2 seconds of rolling

Additionally, a calculation was made of the force at which a drop of water rolls down from an inclined surface. The volume of water droplets was $0.05 \mathrm{ml}$ and was the same for different surfaces. In this case, its mass was determined in accordance with the formula

$$
m=V_{p}
$$

where $\rho$ is the density of water.

Rolling occurs when the back edge of a drop comes off. The drop condition is determined by the expression

$$
F=m q \sin \alpha
$$

where $\mathrm{m}$ is the mass of a drop of water, $\mathrm{kg}$;

$\alpha$ - rolling angle, hail;

$F$-breakout force, $\mathrm{n}$.

The force $\mathrm{F}$ is the sum of the adhesion values of the water droplet and the friction force. Table 2 shows the values of the force $F$ for a hydrophilic and superhydrophobic surface.

Table 2.Angle of rolling off a drop of water from a metal surface

\begin{tabular}{|c|c|c|}
\hline Surface type & Angle of rolling, hail & Breakout force, $\mathbf{n}$ \\
\hline hydrophilic & 31 & $2,55^{*} 10^{-4}$ \\
\hline superhydrophobic & 10 & $0,85^{*} 10-4$ \\
\hline
\end{tabular}

The calculation results show that the breakout force on the superhydrophobic metal surface is 3 times less, which provides easier rolling drop of water from the surface and its anti-icing properties.

One of the characteristics that characterizes the anti-icing properties of superhydrophobic surfaces is the delay in the crystallization of droplets on such surfaces. To assess the kinetics of freezing a drop of water on a superhydrophobic surface, the following experiment was conducted. A drop of water was placed on the superhydrophobic surface, which was placed in a freezer at a temperature of $-18^{\circ} \mathrm{C}$. Studies of the dynamics of freezing drops on the surface were performed using a TESTO 875-1 thermal imager.

Analysis of the experimental data (Table 3) indicates, that in the first freezing stage (up to 15 minutes) the temperature of the surface of the coating is higher than the temperature 
of the water droplet, i.e. heat is transferred from the surface of the coating to a drop of water. On the 18th minute of freezing, the temperature of a water drop becomes $1^{\circ} \mathrm{C}$ lower than the surface temperature of the coating. In the future, the temperature difference increases. Studies have shown that the freezing of a drop of water on a hydrophilic surface (substrate of cement-sand mortar) occurs much earlier and is 21 minutes, while on superhydrophobic - 30 minutes.

Table 3. The temperature of a water droplet during freezing

\begin{tabular}{|c|c|c|}
\hline \multirow{2}{*}{ Time min } & \multicolumn{2}{|c|}{ The temperature, ${ }^{\mathbf{0}} \mathbf{C}$} \\
\cline { 2 - 3 } & water drops & coating surfaces \\
\hline 1 & 20 & 23,5 \\
\hline 3 & 18,4 & 22 \\
\hline 6 & 16,7 & 19,8 \\
\hline 9 & 12,7 & 15,0 \\
\hline 12 & 10,0 & 11,9 \\
\hline 15 & 7,3 & 8,1 \\
\hline 18 & 6,0 & 5,0 \\
\hline 21 & 4,7 & 3,0 \\
\hline 24 & 1,7 & 0,5 \\
\hline 27 & $-0,3$ & $-1,8$ \\
\hline 30 & $-1,0$ & $-2,5$ \\
\hline 33 & $-1,7$ & $-3,0$ \\
\hline 36 & $-1,8$ & $-3,5$ \\
\hline
\end{tabular}

The results of studies of the temperature distribution on the surface of a water droplet indicate that its distribution is uneven. So, after 27 minutes of cooling, the temperature at the top of the droplet surface is $+0.3^{\circ} \mathrm{C}$, below $-0.6^{\circ} \mathrm{C}$, after 30 minutes cooling $-1.0^{\circ} \mathrm{C}$ and $-1.6^{\circ} \mathrm{C}$, respectively, after 33 minutes $--1.4^{\circ} \mathrm{C}$ and $-2,0^{\circ} \mathrm{C}$, respectively.

\section{Conclusions}

It has been established that the force of detachment of a drop on a superhydrophobic metal surface is 3 times less, which ensures easier rolling of a drop of an ox from the surface and its anti-icing properties. The process of freezing a drop of water on a superhydrophobic surface is a multistage process. In the initial period, there is a transfer of heat from the surface to a drop of water. This stage is followed by the process of freezing the drop, which is manifested in the movement of the freezing front from the substrate upwards.

\section{References}

1. L.Boinovich, A.M. Emelyanenko, V.V. Korolev, A.S. Pashinin, Lang., 1659-1668 (2014)

2. J.B.Boreyko, C.P. Collier, ACS nano, 7(2) 1618-1627 (2013)

3. L.Mishchenko, B. Yatton, V. Bahadur et al. ACS nano., 7699-7707 (2010)

4. S.A.Kulinich, M. Farzaneh, Lang., 8854-8856 (2009)

5. G.Momen, R. Jafari, M. Farzaneh, Appl. Sur. Sci., (2015)

6. S. Zheng, Q Fu, W. Hu et al, Mat. Des., 93, 261-270 (2016)

7. L.B.Boinovich, A.M.Emelyanenko, V.K. Ivanov, A.S. Pashinin, Mat. Interf., 2549-2554 (2013)

8. Cao, L., Jones, A.K., Sikka, Lang., 25( 21), 12444-12448 (2009) 
9. S.L.Sinebryukhov, M/V. Sidorova, V.S.Egorkin, P.M.Nedozorov, A.Yu. Ustinov, S.V. Vestn. Fa. East. Bra. Rus. Ac. of Sci., 5, 95-105 (2011) 10. L.B.Boyunovich, Bull. Rus. Acad. Sci., 10-22 (2013)

11.K.K.S.Lau, J.Bico, K.K.B. Teo,M.Chhowalla, G.A.J.Amaratunga, W.I.Milne, G.H.McKinley, K.K.Gleason, Nano Lett., 1701-1705, (2003)

12. V.I. Loganina, S.N. Kislitsyna, K.A. Sergeeva, Bull. PGUAS: constr., sci. and edu., 1, 19-22 (2019)

13. T.M.Schutzius, S. Jung, T. Maitra et al. Lang., 4807-4821 (2014)

14. M.J.Kreder, J. Alvarenga, P. Kim, J. Aizenberg, Nat. Revi. Mat., 15003 (2016)

15. K.R.Khedir, G.K. Kannarpady, C Ryerson, A.S. Birisa. Prog. Org. Co., 112, 304-318 (2017) 